\title{
Search for Large Extra Dimensions in the Production of Jets and Missing Transverse Energy in $p \bar{p}$ Collisions at $\sqrt{s}=1.96 \mathrm{TeV}$
}

A. Abulencia, ${ }^{23}$ D. Acosta, ${ }^{17}$ J. Adelman, ${ }^{13}$ T. Affolder,${ }^{10}$ T. Akimoto, ${ }^{55}$ M. G. Albrow,${ }^{16}$ D. Ambrose,${ }^{16}$ S. Amerio, ${ }^{43}$ D. Amidei ${ }^{34}$ A. Anastassov, ${ }^{52}$ K. Anikeev,${ }^{16}$ A. Annovi, ${ }^{18}$ J. Antos, ${ }^{1}$ M. Aoki, ${ }^{55}$ G. Apollinari, ${ }^{16}$ J.-F. Arguin, ${ }^{33}$

T. Arisawa, ${ }^{57}$ A. Artikov, ${ }^{14}$ W. Ashmanskas, ${ }^{16}$ A. Attal, ${ }^{8}$ F. Azfar, ${ }^{42}$ P. Azzi-Bacchetta, ${ }^{43}$ P. Azzurri, ${ }^{46}$ N. Bacchetta, ${ }^{43}$ H. Bachacou, ${ }^{28}$ W. Badgett, ${ }^{16}$ A. Barbaro-Galtieri, ${ }^{28}$ V. E. Barnes, ${ }^{48}$ B. A. Barnett, ${ }^{24}$ S. Baroiant, ${ }^{7}$ V. Bartsch,${ }^{30}$ G. Bauer, ${ }^{32}$ P.-H. Beachemin, ${ }^{33}$ F. Bedeschi, ${ }^{46}$ S. Behari, ${ }^{24}$ S. Belforte ${ }^{54}$ G. Bellettini, ${ }^{46}$ J. Bellinger, ${ }^{59}$ A. Belloni, ${ }^{32}$ E. Ben-Haim, ${ }^{44}$ D. Benjamin, ${ }^{15}$ A. Beretvas, ${ }^{16}$ J. Beringer ${ }^{28}$ T. Berry, ${ }^{29}$ A. Bhatti ${ }^{50}$ M. Binkley, ${ }^{16}$ D. Bisello, ${ }^{43}$ R. E. Blair, ${ }^{2}$ C. Blocker, ${ }^{6}$ B. Blumenfeld, ${ }^{24}$ A. Bocci, ${ }^{15}$ A. Bodek, ${ }^{49}$ V. Boisvert, ${ }^{49}$ G. Bolla, ${ }^{48}$ A. Bolshov, ${ }^{32}$ D. Bortoletto, ${ }^{48}$ J. Boudreau, ${ }^{47}$ A. Boveia, ${ }^{10}$ B. Brau, ${ }^{10}$ C. Bromberg,${ }^{35}$ E. Brubaker,${ }^{13}$ J. Budagov, ${ }^{14}$ H. S. Budd, ${ }^{49}$ S. Budd,${ }^{23}$ K. Burkett, ${ }^{16}$ G. Busetto,${ }^{43}$ P. Bussey, ${ }^{20}$ K. L. Byrum, ${ }^{2}$ S. Cabrera, ${ }^{15}$ M. Campanelli, ${ }^{19}$ M. Campbell, ${ }^{34}$ F. Canelli, ${ }^{8}$ A. Canepa, ${ }^{48}$ D. Carlsmith,${ }^{59}$ R. Carosi, ${ }^{46}$ S. Carron, ${ }^{15}$ M. Casarsa ${ }^{54}$ A. Castro, ${ }^{5}$ P. Catastini, ${ }^{46}$ D. Cauz,${ }^{54}$ M. Cavalli-Sforza, ${ }^{3}$ A. Cerri, ${ }^{28}$ L. Cerrito, ${ }^{42}$ S. H. Chang, ${ }^{27}$ J. Chapman, ${ }^{34}$ Y. C. Chen, ${ }^{1}$ M. Chertok, ${ }^{7}$ G. Chiarelli, ${ }^{46}$ G. Chlachidze, ${ }^{14}$ F. Chlebana, ${ }^{16}$ I. Cho, ${ }^{27}$ K. Cho, ${ }^{27}$ D. Chokheli, ${ }^{14}$ J. P. Chou, ${ }^{21}$ P. H. Chu, ${ }^{23}$ S. H. Chuang, ${ }^{59}$ K. Chung, ${ }^{12}$ W. H. Chung, ${ }^{59}$ Y. S. Chung, ${ }^{49}$ M. Ciljak, ${ }^{46}$ C. I. Ciobanu, ${ }^{23}$ M. A. Ciocci, ${ }^{46}$ A. Clark, ${ }^{19}$ D. Clark, ${ }^{6}$ M. Coca,${ }^{15}$ G. Compostella, ${ }^{43}$ M. E. Convery, ${ }^{50}$ J. Conway, ${ }^{7}$ B. Cooper ${ }^{30}$ K. Copic,${ }^{34}$ M. Cordelli, ${ }^{18}$ G. Cortiana,${ }^{43}$ F. Cresciolo,${ }^{46}$ A. Cruz,${ }^{17}$ C. Cuenca Almenar, ${ }^{7}$ J. Cuevas, ${ }^{11}$ R. Culbertson, ${ }^{16}$ D. Cyr, ${ }^{59}$ S. DaRonco, ${ }^{43}$ S. D'Auria, ${ }^{20}$ M. D'onofrio, ${ }^{3}$ D. Dagenhart,${ }^{6}$ P. de Barbaro, ${ }^{49}$ S. De Cecco, ${ }^{51}$ A. Deisher ${ }^{28}$ G. De Lentdecker, ${ }^{49}$ M. Dell'Orso, ${ }^{46}$ F. Delli Paoli, ${ }^{43}$ S. Demers, ${ }^{49}$ L. Demortier,${ }^{50}$ J. Deng, ${ }^{15}$ M. Deninno, ${ }^{5}$ D. De Pedis, ${ }^{51}$ P. F. Derwent,${ }^{16}$ C. Dionisi, ${ }^{51}$ J. R. Dittmann, ${ }^{4}$ P. DiTuro, ${ }^{52}$ C. Dörr, ${ }^{25}$ S. Donati, ${ }^{46}$ M. Donega, ${ }^{19}$ P. Dong, ${ }^{8}$ J. Donini, ${ }^{43}$ T. Dorigo, ${ }^{43}$ S. Dube, ${ }^{52}$ K. Ebina, ${ }^{57}$ J. Efron, ${ }^{39}$ J. Ehlers, ${ }^{19}$ R. Erbacher, ${ }^{7}$ D. Errede ${ }^{23}$ S. Errede,${ }^{23}$ R. Eusebi ${ }^{16}$ H. C. Fang, ${ }^{28}$ S. Farrington, ${ }^{29}$ I. Fedorko, ${ }^{46}$ W. T. Fedorko, ${ }^{13}$ R. G. Feild, ${ }^{60}$ M. Feindt, ${ }^{25}$ J. P. Fernandez,${ }^{31}$ R. Field, ${ }^{17}$ G. Flanagan ${ }^{48}$ L. R. Flores-Castillo, ${ }^{47}$ A. Foland,${ }^{21}$ S. Forrester, ${ }^{7}$ G. W. Foster, ${ }^{16}$ M. Franklin, ${ }^{21}$ J. C. Freeman, ${ }^{28}$ I. Furic,,${ }^{13}$ M. Gallinaro, ${ }^{50}$ J. Galyardt, ${ }^{12}$ J. E. Garcia, ${ }^{46}$ M. Garcia Sciveres, ${ }^{28}$ A. F. Garfinkel, ${ }^{48}$ C. Gay, ${ }^{60}$ H. Gerberich, ${ }^{23}$ D. Gerdes,${ }^{34}$ S. Giagu,${ }^{51}$ P. Giannetti, ${ }^{46}$ A. Gibson, ${ }^{28}$ K. Gibson, ${ }^{12}$ C. Ginsburg, ${ }^{16}$ N. Giokaris, ${ }^{14}$ K. Giolo,${ }^{48}$ M. Giordani, ${ }^{54}$ P. Giromini, ${ }^{18}$ M. Giunta, ${ }^{46}$ G. Giurgiu, ${ }^{12}$ V. Glagolev, ${ }^{14}$ D. Glenzinski, ${ }^{16}$ M. Gold,${ }^{37}$ N. Goldschmidt,${ }^{34}$ J. Goldstein, ${ }^{42}$ G. Gomez, ${ }^{11}$ G. Gomez-Ceballos, ${ }^{11}$ M. Goncharov,${ }^{53}$ O. González, ${ }^{31}$ I. Gorelov, ${ }^{37}$ A. T. Goshaw, ${ }^{15}$ Y. Gotra, ${ }^{47}$ K. Goulianos, ${ }^{50}$ A. Gresele, ${ }^{43}$ M. Griffiths, ${ }^{29}$ S. Grinstein, ${ }^{21}$ C. Grosso-Pilcher,${ }^{13}$ R. C. Group,${ }^{17}$ U. Grundler, ${ }^{23}$ J. Guimaraes da Costa, ${ }^{21}$ Z. Gunay-Unalan, ${ }^{35}$ C. Haber ${ }^{28}$ S. R. Hahn, ${ }^{16}$ K. Hahn, ${ }^{45}$ E. Halkiadakis, ${ }^{52}$ A. Hamilton, ${ }^{33}$ B.-Y. Han, ${ }^{49}$ J. Y. Han,${ }^{49}$ R. Handler, ${ }^{59}$ F. Happacher, ${ }^{18}$ K. Hara, ${ }^{55}$ M. Hare, ${ }^{56}$ S. Harper, ${ }^{42}$ R. F. Harr, ${ }^{58}$ R. M. Harris,${ }^{16}$ K. Hatakeyama, ${ }^{50}$ J. Hauser, ${ }^{8}$ C. Hays, ${ }^{15}$ A. Heijboer, ${ }^{45}$ B. Heinemann,${ }^{29}$ J. Heinrich, ${ }^{45}$ M. Herndon, ${ }^{59}$ D. Hidas, ${ }^{15}$ C. S. Hill, ${ }^{10}$ D. Hirschbuehl, ${ }^{25}$ A. Hocker, ${ }^{16}$ A. Holloway, ${ }^{21}$ S. Hou, ${ }^{1}$ M. Houlden, ${ }^{29}$ S.-C. Hsu, ${ }^{9}$ B. T. Huffman,${ }^{42}$ R. E. Hughes, ${ }^{39}$ J. Huston, ${ }^{35}$ J. Incandela, ${ }^{10}$ G. Introzzi, ${ }^{46}$ M. Iori ${ }^{51}$ Y. Ishizawa, ${ }^{55}$ A. Ivanov, ${ }^{7}$ B. Iyutin, ${ }^{32}$ E. James, ${ }^{16}$ D. Jang, ${ }^{52}$ B. Jayatilaka, ${ }^{34}$ D. Jeans,${ }^{51}$ H. Jensen, ${ }^{16}$ E. J. Jeon, ${ }^{27}$ S. Jindariani, ${ }^{17}$ M. Jones,${ }^{48}$ K. K. Joo, ${ }^{27}$ S. Y. Jun, ${ }^{12}$ T. R. Junk, ${ }^{23}$ T. Kamon, ${ }^{53}$ J. Kang, ${ }^{34}$ P.E. Karchin, ${ }^{58}$ Y. Kato, ${ }^{41}$ Y. Kemp,${ }^{25}$ R. Kephart, ${ }^{16}$ U. Kerzel, ${ }^{25}$ V. Khotilovich, ${ }^{53}$ B. Kilminster, ${ }^{39}$ D. H. Kim, ${ }^{27}$ H. S. Kim,${ }^{27}$ J.E. Kim, ${ }^{27}$ M. J. Kim, ${ }^{12}$ S. B. Kim, ${ }^{27}$ S. H. Kim ${ }^{55}$ Y. K. Kim, ${ }^{13}$ L. Kirsch, ${ }^{6}$ S. Klimenko, ${ }^{17}$ M. Klute, ${ }^{32}$ B. Knuteson, ${ }^{32}$ B. R. Ko, ${ }^{15}$ H. Kobayashi, ${ }^{55}$ K. Kondo, ${ }^{57}$ D. J. Kong, ${ }^{27}$ J. Konigsberg, ${ }^{17}$ A. Korytov, ${ }^{17}$ A. V. Kotwal, ${ }^{15}$ A. Kovalev, ${ }^{45}$ A. Kraan, ${ }^{45}$ J. Kraus, ${ }^{23}$ I. Kravchenko, ${ }^{32}$ M. Kreps,${ }^{25}$ J. Kroll, ${ }^{45}$ N. Krumnack, ${ }^{4}$ M. Kruse, ${ }^{15}$ V. Krutelyov, ${ }^{53}$ S.E. Kuhlmann, ${ }^{2}$ Y. Kusakabe, ${ }^{57}$ S. Kwang, ${ }^{13}$ A. T. Laasanen, ${ }^{48}$ S. Lai, ${ }^{33}$ S. Lami, ${ }^{46}$ S. Lammel, ${ }^{16}$ M. Lancaster, ${ }^{30}$ R. L. Lander, ${ }^{7}$ K. Lannon, ${ }^{39}$ A. Lath,${ }^{52}$ G. Latino, ${ }^{46}$ I. Lazzizzera, ${ }^{43}$ T. LeCompte, ${ }^{2}$ J. Lee, ${ }^{49}$ J. Lee, ${ }^{27}$ Y. J. Lee, ${ }^{27}$ S. W. Lee,${ }^{53}$ R. Lefèvre, ${ }^{3}$ N. Leonardo, ${ }^{32}$ S. Leone, ${ }^{46}$ S. Levy, ${ }^{13}$ J. D. Lewis, ${ }^{16}$ C. Lin, ${ }^{60}$ C. S. Lin, ${ }^{16}$ M. Lindgren, ${ }^{16}$ E. Lipeles, ${ }^{9}$ T. M. Liss, ${ }^{23}$ A. Lister, ${ }^{19}$ D. O. Litvintsev, ${ }^{16}$ T. Liu, ${ }^{16}$ N. S. Lockyer, ${ }^{45}$ A. Loginov, ${ }^{36}$ M. Loreti, ${ }^{43}$ P. Loverre, ${ }^{51}$ R.-S. Lu, ${ }^{1}$ D. Lucchesi,${ }^{43}$ P. Lujan, ${ }^{28}$ P. Lukens, ${ }^{16}$ G. Lungu, ${ }^{17}$ L. Lyons, ${ }^{42}$ J. Lys, ${ }^{28}$ R. Lysak, ${ }^{1}$ E. Lytken ${ }^{48}$ P. Mack, ${ }^{25}$ D. MacQueen, ${ }^{33}$ R. Madrak, ${ }^{16}$ K. Maeshima, ${ }^{16}$ T. Maki, ${ }^{22}$ P. Maksimovic, ${ }^{24}$ S. Malde, ${ }^{42}$ G. Manca, ${ }^{29}$ F. Margaroli, ${ }^{5}$ R. Marginean, ${ }^{16}$ C. Marino, ${ }^{23}$ A. Martin, ${ }^{60}$ V. Martin, ${ }^{38}$ M. Martínez, ${ }^{3}$ T. Maruyama, ${ }^{55}$ H. Matsunaga,${ }^{55}$ M. E. Mattson, ${ }^{58}$ R. Mazini,${ }^{33}$ P. Mazzanti, ${ }^{5}$ K. S. McFarland, ${ }^{49}$ P. McIntyre, ${ }^{53}$ R. McNulty, ${ }^{29}$ A. Mehta, ${ }^{29}$ S. Menzemer, ${ }^{11}$ A. Menzione, ${ }^{46}$ P. Merkel,${ }^{48}$ C. Mesropian, ${ }^{50}$ A. Messina, ${ }^{51}$ M. von der Mey, ${ }^{8}$ T. Miao, ${ }^{16}$ N. Miladinovic,${ }^{6}$ J. Miles,${ }^{32}$ R. Miller, ${ }^{35}$ J. S. Miller ${ }^{34}$ C. Mills, ${ }^{10}$ M. Milnik,${ }^{25}$ R. Miquel, ${ }^{28}$ A. Mitra, ${ }^{1}$ G. Mitselmakher, ${ }^{17}$ A. Miyamoto, ${ }^{26}$ N. Moggi, ${ }^{5}$ B. Mohr,${ }^{8}$ R. Moore, ${ }^{16}$ M. Morello, ${ }^{46}$ P. Movilla Fernandez, ${ }^{28}$ J. Mülmenstädt, ${ }^{28}$ A. Mukherjee,${ }^{16}$ Th. Muller ${ }^{25}$ R. Mumford,${ }^{24}$ P. Murat, ${ }^{16}$ J. Nachtman, ${ }^{16}$ J. Naganoma, ${ }^{57}$ S. Nahn, ${ }^{32}$ I. Nakano, ${ }^{40}$ A. Napier, ${ }^{56}$ D. Naumov, ${ }^{37}$ V. Necula, ${ }^{17}$ 
C. Neu, ${ }^{45}$ M. S. Neubauer, ${ }^{9}$ J. Nielsen, ${ }^{28}$ T. Nigmanov ${ }^{47}$ L. Nodulman, ${ }^{2}$ O. Norniella, ${ }^{3}$ E. Nurse,${ }^{30}$ T. Ogawa,${ }^{57}$ S. H. Oh, ${ }^{15}$ Y.D. Oh, ${ }^{27}$ T. Okusawa ${ }^{41}$ R. Oldeman,${ }^{29}$ R. Orava, ${ }^{22}$ K. Osterberg, ${ }^{22}$ C. Pagliarone, ${ }^{46}$ E. Palencia, ${ }^{11}$ R. Paoletti, ${ }^{46}$ V. Papadimitriou, ${ }^{16}$ A. A. Paramonov,${ }^{13}$ B. Parks, ${ }^{39}$ S. Pashapour, ${ }^{33}$ J. Patrick,,${ }^{16}$ G. Pauletta,${ }^{54}$ M. Paulini, ${ }^{12}$ C. Paus, ${ }^{32}$ D. E. Pellett, ${ }^{7}$ A. Penzo,${ }^{54}$ T. J. Phillips, ${ }^{15}$ G. Piacentino, ${ }^{46}$ J. Piedra, ${ }^{44}$ L. Pinera,${ }^{17}$ K. Pitts, ${ }^{23}$ C. Plager,${ }^{8}$ L. Pondrom,${ }^{59}$ X. Portell, ${ }^{3}$ O. Poukhov, ${ }^{14}$ N. Pounder, ${ }^{42}$ F. Prakoshyn, ${ }^{14}$ A. Pronko, ${ }^{16}$ J. Proudfoot, ${ }^{2}$ F. Ptohos, ${ }^{18}$ G. Punzi,${ }^{46}$ J. Pursley, ${ }^{42}$ J. Rademacker, ${ }^{42}$ A. Rahaman, ${ }^{47}$ A. Rakitin, ${ }^{32}$ S. Rappoccio, ${ }^{21}$ F. Ratnikov, ${ }^{52}$ B. Reisert, ${ }^{16}$ V. Rekovic,${ }^{37}$ N. van Remortel, ${ }^{22}$ P. Renton, ${ }^{42}$ M. Rescigno, ${ }^{51}$ S. Richter, ${ }^{25}$ F. Rimondi, ${ }^{5}$ L. Ristori, ${ }^{46}$ W. J. Robertson, ${ }^{15}$ A. Robson, ${ }^{20}$ T. Rodrigo, ${ }^{11}$ E. Rogers,${ }^{23}$ S. Rolli, ${ }^{56}$ R. Roser, ${ }^{16}$ M. Rossi,${ }^{54}$ R. Rossin,,${ }^{17}$ C. Rott,${ }^{48}$ A. Ruiz, ${ }^{11}$ J. Russ, ${ }^{12}$ V. Rusu, ${ }^{13}$ H. Saarikko, ${ }^{22}$ S. Sabik, ${ }^{33}$ A. Safonov,${ }^{53}$ W. K. Sakumoto, ${ }^{49}$ G. Salamanna, ${ }^{51}$ O. Saltó, ${ }^{3}$ D. Saltzberg, ${ }^{8}$ C. Sanchez, ${ }^{3}$ L. Santi, ${ }^{54}$ S. Sarkar, ${ }^{51}$ L. Sartori, ${ }^{46}$ K. Sato ${ }^{55}$ P. Savard, ${ }^{33}$ A. Savoy-Navarro, ${ }^{44}$ T. Scheidle, ${ }^{25}$ P. Schlabach, ${ }^{16}$

E. E. Schmidt, ${ }^{16}$ M. P. Schmidt, ${ }^{60}$ M. Schmitt, ${ }^{38}$ T. Schwarz, ${ }^{34}$ L. Scodellaro, ${ }^{11}$ A. L. Scott, ${ }^{10}$ A. Scribano, ${ }^{46}$ F. Scuri ${ }^{46}$ A. Sedov, ${ }^{48}$ S. Seidel,${ }^{37}$ Y. Seiya, ${ }^{41}$ A. Semenov, ${ }^{14}$ L. Sexton-Kennedy, ${ }^{16}$ I. Sfiligoi, ${ }^{18}$ M. D. Shapiro ${ }^{28}$ T. Shears, ${ }^{29}$ P. F. Shepard ${ }^{47}$ D. Sherman, ${ }^{21}$ M. Shimojima,${ }^{55}$ M. Shochet,${ }^{13}$ Y. Shon, ${ }^{59}$ I. Shreyber, ${ }^{36}$ A. Sidoti, ${ }^{44}$ P. Sinervo, ${ }^{33}$ A. Sisakyan, ${ }^{14}$ J. Sjolin, ${ }^{42}$ A. Skiba,${ }^{25}$ A. J. Slaughter,${ }^{16}$ K. Sliwa,${ }^{56}$ J. R. Smith, ${ }^{7}$ F. D. Snider,${ }^{16}$ R. Snihur, ${ }^{33}$ M. Soderberg, ${ }^{34}$ A. Soha,${ }^{7}$ S. Somalwar, ${ }^{52}$ V. Sorin,${ }^{35}$ J. Spalding, ${ }^{16}$ M. Spezziga, ${ }^{16}$ F. Spinella, ${ }^{46}$ T. Spreitzer,${ }^{33}$ P. Squillacioti, ${ }^{46}$ M. Stanitzki, ${ }^{60}$ A. Staveris-Polykalas, ${ }^{46}$ R. St. Denis,${ }^{20}$ B. Stelzer, ${ }^{8}$ O. Stelzer-Chilton, ${ }^{42}$ D. Stentz, ${ }^{38}$ J. Strologas,${ }^{37}$ D. Stuart, ${ }^{10}$ J. S. Suh,${ }^{27}$ A. Sukhanov, ${ }^{17}$ K. Sumorok, ${ }^{32}$ H. Sun, ${ }^{56}$ T. Suzuki,${ }^{55}$ A. Taffard,${ }^{23}$ R. Takashima, ${ }^{40}$ Y. Takeuchi, ${ }^{55}$ K. Takikawa, ${ }^{55}$ M. Tanaka, ${ }^{2}$ R. Tanaka, ${ }^{40}$ N. Tanimoto, ${ }^{40}$ M. Tecchio, ${ }^{34}$ P. K. Teng, ${ }^{1}$ K. Terashi, ${ }^{50}$ S. Tether, ${ }^{32}$ J. Thom, ${ }^{16}$ A. S. Thompson, ${ }^{16}$ E. Thomson,,${ }^{45}$ P. Tipton, ${ }^{49}$ V. Tiwari,${ }^{12}$ S. Tkaczyk,${ }^{16}$ D. Toback,${ }^{53}$ S. Tokar, ${ }^{14}$ K. Tollefson, ${ }^{35}$ T. Tomura, ${ }^{55} \mathrm{D}$. Tonelli,${ }^{46} \mathrm{M}$. Tönnesmann, ${ }^{35} \mathrm{~S}$. Torre, ${ }^{18} \mathrm{D}$. Torretta, ${ }^{16} \mathrm{~S}$. Tourneur, ${ }^{44} \mathrm{~W}$. Trischuk ${ }^{33}$ R. Tsuchiya, ${ }^{57}$ S. Tsuno,${ }^{40}$ N. Turini, ${ }^{46}$ F. Ukegawa ${ }^{55}$ T. Unverhau ${ }^{20}{ }^{20}$ S. Uozumi,${ }^{55}$ D. Usynin,${ }^{45}$ A. Vaiciulis, ${ }^{49}$ S. Vallecorsa, ${ }^{19}$ A. Varganov, ${ }^{34}$ E. Vataga,${ }^{37} \mathrm{G}$. Velev, ${ }^{16} \mathrm{G}$. Veramendi, ${ }^{23} \mathrm{~V}$. Veszpremi, ${ }^{48} \mathrm{R}$. Vidal,${ }^{16} \mathrm{I}$. Vila, ${ }^{11} \mathrm{R}$. Vilar, ${ }^{11}$

T. Vine, ${ }^{30}$ I. Vollrath, ${ }^{33}$ I. Volobouev, ${ }^{28}$ G. Volpi, ${ }^{46}$ F. Würthwein, ${ }^{9}$ P. Wagner, ${ }^{53}$ R. G. Wagner, ${ }^{2}$ R. L. Wagner, ${ }^{16}$ W. Wagner ${ }^{25}$ R. Wallny, ${ }^{8}$ T. Walter, ${ }^{25}$ Z. Wan, ${ }^{52}$ S. M. Wang, ${ }^{1}$ A. Warburton, ${ }^{33}$ S. Waschke, ${ }^{20}$ D. Waters,${ }^{30}$

W. C. Wester III, ${ }^{16}$ B. Whitehouse, ${ }^{56}$ D. Whiteson, ${ }^{45}$ A. B. Wicklund, ${ }^{2}$ E. Wicklund, ${ }^{16}$ G. Williams,${ }^{33}$ H. H. Williams,${ }^{45}$ P. Wilson, ${ }^{16}$ B. L. Winer, ${ }^{39}$ P. Wittich, ${ }^{16}$ S. Wolbers, ${ }^{16}$ C. Wolfe, ${ }^{13}$ T. Wright,${ }^{34}$ X. Wu, ${ }^{19}$ S. M. Wynne, ${ }^{29}$ A. Yagil,${ }^{16}$ K. Yamamoto ${ }^{41}$ J. Yamaoka, ${ }^{52}$ T. Yamashita, ${ }^{40}$ C. Yang, ${ }^{60}$ U. K. Yang, ${ }^{13}$ W. M. Yao, ${ }^{28}$ G. P. Yeh, ${ }^{16}$ J. Yoh, ${ }^{16}$ K. Yorita, ${ }^{13}$ T. Yoshida, ${ }^{41}$ G. B. Yu, ${ }^{49}$ I. Yu, ${ }^{27}$ S. S. Yu, ${ }^{16}$ J. C. Yun, ${ }^{16}$ L. Zanello, ${ }^{51}$ A. Zanetti, ${ }^{54}$ I. Zaw, ${ }^{21}$ F. Zetti, ${ }^{46}$ X. Zhang, ${ }^{23}$ J. Zhou, ${ }^{52}$ and S. Zucchelli ${ }^{5}$

\title{
(CDF Collaboration)
}

\author{
${ }^{1}$ Institute of Physics, Academia Sinica, Taipei, Taiwan 11529, Republic of China \\ ${ }^{2}$ Argonne National Laboratory, Argonne, Illinois 60439, USA \\ ${ }^{3}$ Institut de Fisica d'Altes Energies, Universitat Autonoma de Barcelona, E-08193, Bellaterra (Barcelona), Spain \\ ${ }^{4}$ Baylor University, Waco, Texas 76798, USA \\ ${ }^{5}$ Istituto Nazionale di Fisica Nucleare, University of Bologna, I-40127 Bologna, Italy \\ ${ }^{6}$ Brandeis University, Waltham, Massachusetts 02254, USA \\ ${ }^{7}$ University of California, Davis, Davis, California 95616, USA \\ ${ }^{8}$ University of California, Los Angeles, Los Angeles, California 90024, USA \\ ${ }^{9}$ University of California, San Diego, La Jolla, California 92093, USA \\ ${ }^{10}$ University of California, Santa Barbara, Santa Barbara, California 93106, USA \\ ${ }^{11}$ Instituto de Fisica de Cantabria, CSIC-University of Cantabria, 39005 Santander, Spain \\ ${ }^{12}$ Carnegie Mellon University, Pittsburgh, Pennsylvania 15213, USA \\ ${ }^{13}$ Enrico Fermi Institute, University of Chicago, Chicago, Illinois 60637, USA \\ ${ }^{14}$ Joint Institute for Nuclear Research, RU-141980 Dubna, Russia \\ ${ }^{15}$ Duke University, Durham, North Carolina 27708 \\ ${ }^{16}$ Fermi National Accelerator Laboratory, Batavia, Illinois 60510, USA \\ ${ }^{17}$ University of Florida, Gainesville, Florida 32611, USA \\ ${ }^{18}$ Laboratori Nazionali di Frascati, Istituto Nazionale di Fisica Nucleare, I-00044 Frascati, Italy \\ ${ }^{19}$ University of Geneva, CH-1211 Geneva 4, Switzerland \\ ${ }^{20}$ Glasgow University, Glasgow G12 8QQ, United Kingdom \\ ${ }^{21}$ Harvard University, Cambridge, Massachusetts 02138, USA \\ ${ }^{22}$ Division of High Energy Physics, Department of Physics, University of Helsinki and Helsinki Institute of Physics, \\ FIN-00014, Helsinki, Finland
}




\author{
${ }^{23}$ University of Illinois, Urbana, Illinois 61801, USA \\ ${ }^{24}$ The Johns Hopkins University, Baltimore, Maryland 21218, USA \\ ${ }^{25}$ Institut für Experimentelle Kernphysik, Universität Karlsruhe, 76128 Karlsruhe, Germany \\ ${ }^{26}$ High Energy Accelerator Research Organization (KEK), Tsukuba, Ibaraki 305, Japan \\ ${ }^{27}$ Center for High Energy Physics: Kyungpook National University, Taegu 702-701, Korea; \\ Seoul National University, Seoul 151-742, Korea, \\ and SungKyunKwan University, Suwon 440-746, Korea \\ ${ }^{28}$ Ernest Orlando Lawrence Berkeley National Laboratory, Berkeley, California 94720, USA \\ ${ }^{29}$ University of Liverpool, Liverpool L69 7ZE, United Kingdom \\ ${ }^{30}$ University College London, London WC1E 6BT, United Kingdom \\ ${ }^{31}$ Centro de Investigaciones Energeticas Medioambientales y Tecnologicas, E-28040 Madrid, Spain \\ ${ }^{32}$ Massachusetts Institute of Technology, Cambridge, Massachusetts 02139, USA \\ ${ }^{33}$ Institute of Particle Physics: McGill University, Montréal, Canada H3A 2T8, \\ and University of Toronto, Toronto, Canada M5S 1A7 \\ ${ }^{34}$ University of Michigan, Ann Arbor, Michigan 48109, USA \\ ${ }^{35}$ Michigan State University, East Lansing, Michigan 48824, USA \\ ${ }^{36}$ Institution for Theoretical and Experimental Physics, ITEP, Moscow 117259, Russia \\ ${ }^{37}$ University of New Mexico, Albuquerque, New Mexico 87131, USA \\ ${ }^{38}$ Northwestern University, Evanston, Illinois 60208, USA \\ ${ }^{39}$ The Ohio State University, Columbus, Ohio 43210, USA \\ ${ }^{40}$ Okayama University, Okayama 700-8530, Japan \\ ${ }^{41}$ Osaka City University, Osaka 588, Japan \\ ${ }^{42}$ University of Oxford, Oxford OX1 3RH, United Kingdom \\ ${ }^{43}$ University of Padova, Istituto Nazionale di Fisica Nucleare, Sezione di Padova-Trento, I-35131 Padova, Italy \\ ${ }^{44}$ LPNHE, Universite Pierre et Marie Curie/IN2P3-CNRS, UMR7585, Paris F-75252, France \\ ${ }^{45}$ University of Pennsylvania, Philadelphia, Pennsylvania 19104, USA \\ ${ }^{46}$ Istituto Nazionale di Fisica Nucleare Pisa, Universities of Pisa, Siena and Scuola Normale Superiore, I-56127 Pisa, Italy \\ ${ }^{47}$ University of Pittsburgh, Pittsburgh, Pennsylvania 15260, USA \\ ${ }^{48}$ Purdue University, West Lafayette, Indiana 47907, USA \\ ${ }^{49}$ University of Rochester, Rochester, New York 14627, USA \\ ${ }^{50}$ The Rockefeller University, New York, New York 10021, USA \\ ${ }^{51}$ Istituto Nazionale di Fisica Nucleare, Sezione di Roma 1, University of Rome "La Sapienza," I-00185 Roma, Italy \\ ${ }^{52}$ Rutgers University, Piscataway, New Jersey 08855, USA \\ ${ }^{53}$ Texas A\&M University, College Station, Texas 77843, USA \\ ${ }^{54}$ Istituto Nazionale di Fisica Nucleare, University of Trieste/Udine, Italy \\ ${ }^{55}$ University of Tsukuba, Tsukuba, Ibaraki 305, Japan \\ ${ }^{56}$ Tufts University, Medford, Massachusetts 02155, USA \\ ${ }^{57}$ Waseda University, Tokyo 169, Japan \\ ${ }^{58}$ Wayne State University, Detroit, Michigan 48201, USA \\ ${ }^{59}$ University of Wisconsin, Madison, Wisconsin 53706, USA \\ ${ }^{60}$ Yale University, New Haven, Connecticut 06520, USA \\ (Received 10 April 2006; published 24 October 2006)
}

We present the results of a search for new physics in the jets plus missing transverse energy data sample collected from $368 \mathrm{pb}^{-1}$ of $p \bar{p}$ collisions at $\sqrt{s}=1.96 \mathrm{TeV}$ recorded by the Collider Detector at Fermilab. We compare the number of events observed in the data with a data-based estimate of the standard model backgrounds contributing to this signature. We observe no significant excess of events, and we interpret this null result in terms of lower limits on the fundamental Planck scale for a large extra dimensions scenario.

DOI: 10.1103/PhysRevLett.97.171802

One of the simplest new physics signatures that can be explored at a hadron collider consists of a very energetic jet and large missing transverse energy $\left(\boldsymbol{E}_{T}\right)$. The Tevatron offers a unique opportunity to explore energy regimes that could yield new physics that have not been accessible at previous colliders. We take advantage of this opportunity by performing a signature-based, high energy monojet search. While a wide range of exotic physics both known
PACS numbers: $13.85 . \mathrm{Rm}, 04.50 .+\mathrm{h}, 11.25 . \mathrm{Wx}, 14.80 .-\mathrm{j}$

and not yet imagined could yield such a signature, the most exciting recent scenario involves large extra dimensions (LED) [1]. LED are an essential ingredient of proposed solutions to the most fundamental problems of physics including the hierarchy problem [2] and the observed value of the dark energy [3].

In LED scenarios, gravitons or their superpartners [3,4] are responsible for the observed $\mathscr{E}_{T}$. In the simplest $2 \rightarrow 2$ 
tree-level processes possible at a hadron collider experiment, any of the gravitational states (we denote all possible states with spins from 0 to 2 by $G$ ) can be directly produced in processes such as $q \bar{q} \rightarrow g G, q g \rightarrow q G$, and $g g \rightarrow g G$, leaving the final state quark $(q)$ or gluon $(g)$ to produce a single jet [1].

Graviton emission is within reach of the Tevatron provided that the $(4+n)$-dimensional Planck scale $\left(M_{D}\right)$ is around $1 \mathrm{TeV}[1,2]$. This can be the case if the radii $R$ of the $n$ compactified extra dimensions are sufficiently large. Assuming $n$ extra dimensions of the same size, the relationship between the four-dimensional effective Planck scale $M_{\mathrm{Pl}} \sim 10^{19} \mathrm{GeV}$ and the fundamental Planck scale $M_{D}$ is given by the generalized Gauss theorem [2]:

$$
M_{\mathrm{Pl}}^{2}=8 \pi R^{n} M_{D}^{2+n} .
$$

In the absence of a significant excess of events relative to the background expectations, lower limits can be set on $M_{D}$, a fundamental parameter common to all LED models.

Monojet searches performed in run I [5] at the Tevatron were consistent with standard model (SM) expectations. In this Letter we present a new search using $368 \mathrm{pb}^{-1}$ of data, recorded by the Collider Detector at Fermilab (CDF) in run II of the Tevatron. The sensitivity of this analysis to new physics in general and to LED scenarios, in particular, is significantly improved by the increase in center-of-mass energy for run II $p \bar{p}$ collisions (1.80 to $1.96 \mathrm{TeV}$ ), an improved CDF detector, and a factor of 4 increase in the integrated luminosity over the data sample used in run I.

A complete description of the CDF II detector is given in Refs. [6]. The important components used in the reconstruction of the events for this analysis include a tracking system consisting of a silicon-strip vertex detector surrounded by an open-cell drift chamber. The tracking system is situated within a $1.4 \mathrm{~T}$ solenoidal magnetic field to measure charged particle momenta transverse to the beamline $\left(p_{T}\right)$. Outside the magnet, scintillator-based electromagnetic and hadronic calorimeter modules are arranged in projective tower geometries to reconstruct the energy and direction of the particle jets. The outermost detection system consists of planes of multilayered drift chambers for detecting muons.

We select events in the trigger system based on the presence of a jet with transverse energy $E_{T}>100 \mathrm{GeV}$ and further require offline that the highest $E_{T}$ jet in the event (the leading jet) has $E_{T}>150 \mathrm{GeV}$. With an offline threshold of $150 \mathrm{GeV}$, the jet trigger is $>99 \%$ efficient for the events in our sample. Jets are reconstructed using a fixed cone algorithm with cone size $\Delta \mathcal{R} \equiv$ $\sqrt{(\Delta \eta)^{2}+(\bar{\phi} \phi)^{2}}=0.7$ [7] over the full pseudorapidity coverage of the calorimeters $(|\eta|<3.6)$. The jet $E_{T}$ is corrected to account for the effects of fragmentation, calorimeter nonuniformities, and energy from the rest of the event [8]. To increase the acceptance for events containing a second jet originating from quark or gluon radiation, we accept events that contain a second jet with $E_{T}<60 \mathrm{GeV}$. However, we reject events that contain three or more reconstructed jets with $E_{T}>20 \mathrm{GeV}$ because these events have a much lower signal-to-background ratio than one-jet and two-jet events. Since the $\mathbb{E}_{T}$ [9] for most backgrounds is typically much lower than that of the signal, we require $\mathscr{E}_{T}>120 \mathrm{GeV}$. This value is set lower than the jet $E_{T}$ threshold of $150 \mathrm{GeV}$ to keep the signal efficiency high given the $\not E_{T}$ resolution.

To further reduce backgrounds from multijet events in which one or more jets are badly mismeasured, we require that the azimuth $\phi$ of the observed $\not_{T}$ is separated by more than 0.3 radians from the $\phi$ of any second jet. To eliminate noncollision background events originating from beam halo, cosmic rays, and detector noise, we require that the leading jet is central $(|\eta|<1.0)$ and contained within the instrumented parts of the calorimeter. Since a jet with $|\eta|<1.0$ is within the fiducial tracking volume, we can search for tracks pointing towards the region of the calorimeter in which the jet is found. For all jets within the fiducial tracking volume, we require at least two associated tracks whose $p_{T}$ add up to at least $10 \%$ of the jet $E_{T}$. We also require an event vertex reconstructed from six or more tracks that is within $60 \mathrm{~cm}$ of the detector center in $z$ (the coordinate parallel to the colliding beams). For the leading, central jet we require that the associated tracks used in the $p_{T}$ sum described above are consistent with having originated from this event vertex. To eliminate background from muons produced upstream of the detector that interact and look like jets in the hadronic calorimeter, we require that for each event the total electromagnetic energy of all jets with $E_{T}>20 \mathrm{GeV}$ is at least $10 \%$ of the total $E_{T}$.

The resulting candidate sample contains a significant number of events originating from SM processes which can produce large $\mathscr{E}_{T}$ in the detector. The $\mathscr{E}_{T}$ associated with these processes can originate from either neutrinos in the final state (real $\mathbb{E}_{T}$ ) or other particles that pass into uninstrumented regions of the detector (fake $\mathbb{E}_{T}$ ). The largest SM background is $Z+$ jets where the $Z$ boson subsequently decays into neutrinos $(Z \rightarrow \nu \bar{\nu})$. This background has the same event topology as our signal and is thus irreducible. The next most significant SM background comes from $W(\rightarrow l \nu)+$ jets production $(l=e, \mu$, or $\tau)$ where the lepton is unidentified. The contribution of this background is suppressed by rejecting events that contain an isolated track with $p_{T}>10 \mathrm{GeV}$ (a potential muon) or a jet with $E_{T}>20 \mathrm{GeV}$ for which the electromagnetic energy fraction is above $90 \%$ (a potential electron). Track isolation is defined using the measured energy in the calorimeter within a $\Delta \mathcal{R}<0.4$ cone around the reconstructed track, after subtracting the measured energy in those calorimeter towers intersected by the track. Tracks are defined to be isolated if this energy is less than $10 \%$ of the measured track $p_{T}$. We refer to the $W / Z+$ jets backgrounds collectively as electroweak backgrounds. 
The number of electroweak background events in the candidate sample is estimated by measuring cross sections for $Z(\rightarrow l l)+$ jets and $W(\rightarrow l \nu)+$ jets $(l=e$ or $\mu)$ production from independent data samples collected using high $E_{T}$ single electron and high $p_{T}$ single muon triggers. We select events that contain a muon with $p_{T}>20 \mathrm{GeV}$ or an electron with $E_{T}>25 \mathrm{GeV}$ using standard lepton selection criteria [10] to construct a low-background sample of lepton candidates. Starting from this sample, we select $W \rightarrow l \nu$ candidates by requiring $\not E_{T}>25 \mathrm{GeV}\left(\not \mathbb{E}_{T}>\right.$ $20 \mathrm{GeV}$ for muon events) and $Z \rightarrow l l$ candidates by requiring a second lepton that satisfies a looser set of selection criteria [10]. The cosmic ray background in both candidate samples is reduced by rejecting events in which tracks passing through opposite hemispheres of the detector can be reconstructed along a common trajectory.

Using the measured $Z(\rightarrow l l)+$ jets cross sections and the difference in $Z$ branching fractions for charged leptons and neutrinos, we estimate the expected number of $Z(\rightarrow \nu \bar{\nu})+$ jets events in our candidate sample. A second, independent estimate of this background is obtained from the measured $W(\rightarrow l \nu)+$ jets cross sections. In this case we first divide the measured cross section by a theoretical prediction for $R_{(W / Z)}$, the ratio of the $W+$ jets and $Z+$ jets production cross sections and then correct the extrapolated $Z(\rightarrow l l)+$ jets cross section for the $Z$ branching fraction to neutrinos. A slightly more precise prediction for the expected background is obtained by combining the estimates. This combination reduces the statistical uncertainty from $11 \%$ to $10 \%$.

As a consistency check of the event selection, we measure inclusive cross sections for $W / Z$ production and find them to be consistent with published run II measurements [10]. In addition, we measure $W / Z+$ jet production cross sections where the jet criteria are identical to those used in the final selection of our jet plus $\mathscr{E}_{T}$ sample, except that we vary the leading jet $E_{T}$ cut, using thresholds of $60,90,120$, and $150 \mathrm{GeV}$. The larger statistics in the samples obtained using lower jet $E_{T}$ thresholds allow statistically significant comparisons between independent measurements in the electron and muon channels. The observed agreement provides further validation of the cross section measurements made for the $150 \mathrm{GeV}$ leading jet threshold that are used to estimate the electroweak backgrounds in our final candidate sample.

The acceptance values used in the $W / Z+$ jets cross section measurements are obtained from simulated PYTHIA [11] event samples using a full detector simulation based on GEANT3 [12] and corrected to account for measured differences in lepton selection criteria observed in data and simulation. The measured cross sections are shown in Fig. 1. We combine cross section measurements from the electron and muon samples using the default cut of $150 \mathrm{GeV}$ on the $E_{T}$ of the leading jet and obtain $\sigma(W(\rightarrow l \nu)+$ jets $)=0.46 \pm 0.05 \mathrm{pb}$ and $\sigma(Z(\rightarrow l l)+$ jets) $=0.08 \pm 0.02 \mathrm{pb}$.

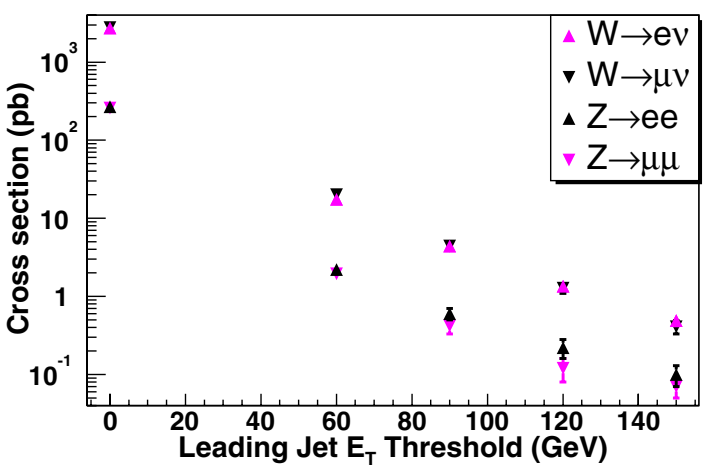

FIG. 1 (color online). $\quad W$ and $Z$ Boson cross sections as a function of leading jet $E_{T}$.

A next-to-leading-order calculation based on the MCFM generator [13] was used to determine the value of $R_{(W / Z)}$. At $P_{T}^{\min }=150 \mathrm{GeV}$, the calculated value of $R_{(W / Z)}$ is $8.15 \pm 0.40$. Based on the combined $Z(\rightarrow l l)+$ jets and $W(\rightarrow l \nu)+$ jets cross section measurements, we estimate $177 \pm 44$ and $125 \pm 15$ background events from $Z(\rightarrow \nu \bar{\nu})+$ jets in our jet plus $\mathbb{E}_{T}$ candidate sample. The two independent results are combined to obtain a final estimate of $130 \pm 14$ events. Estimates for the contributions of the other electroweak backgrounds to the candidate sample are also obtained from the measured $W / Z+$ jets cross sections. We extract, for example, the number of $W(\rightarrow l \nu)+$ jets background events from the measured $W(\rightarrow l \nu)+$ jets cross section based on the percentage of simulated $W(\rightarrow l \nu)+$ jets events which pass our selection criteria. Since the same measured cross sections are used to estimate the contributions of each electroweak background shown in Table I, the uncertainties on these background predictions are fully correlated.

As described previously, events originating from quantum chromodynamics (QCD) multijet production processes can enter our sample when the mismeasurement of one or more jets creates large $\mathbb{E}_{T}$ in the detector. The dominant topology is two-jet events where the second jet is not found by the jet-finding algorithm. To estimate the background contribution of such events, we study dijet

TABLE I. Summary of estimated background contributions and number of events observed for the candidate sample.

\begin{tabular}{lc}
\hline \hline Background & Events \\
\hline$Z \rightarrow \nu \bar{\nu}$ & $130 \pm 14$ \\
$W \rightarrow \tau \nu$ & $60 \pm 7$ \\
$W \rightarrow \mu \nu$ & $36 \pm 4$ \\
$W \rightarrow e \nu$ & $17 \pm 2$ \\
$Z \rightarrow l l$ & $3 \pm 1$ \\
QCD multijet & $15 \pm 10$ \\
Noncollision & $4 \pm 4$ \\
Total expected & $265 \pm 30$ \\
Data observed & 263 \\
\hline \hline
\end{tabular}




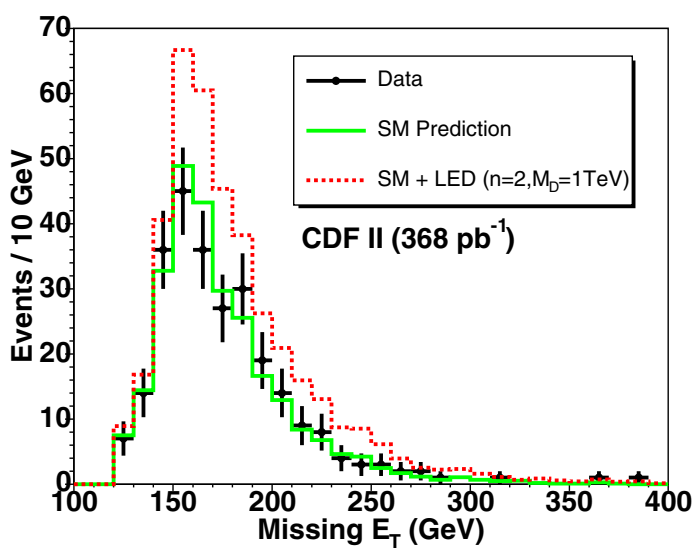

FIG. 2 (color online). Comparison of the event $\not{E}_{T}$ distribution for the 263 events in our candidate sample with the predicted SM distribution. The expected additional contribution from an LED signal for $n=2$ and $M_{D}=1 \mathrm{TeV}$ is plotted on top of the predicted SM distribution.

events in data for which the observed $\not{E}_{T}$ points in the direction of the less energetic jet. We perform a linear extrapolation of the $E_{T}$ distribution for this less energetic jet into the region where the $E_{T}$ drops below our threshold $(20 \mathrm{GeV})$ for defining jets. Monte Carlo studies indicate that an additional relative contribution of approximately $15 \%$ from three-jet events should be added to the number extracted from this method, resulting in an estimate of $15 \pm 10$ background events from QCD multijet production in our final candidate sample. Using timing information from the hadronic calorimeter we estimate the noncollision background, from sources such as cosmic rays, to be $4 \pm 4$ events.

A summary of the estimated background contributions is shown in Table I. We predict a total background of $265 \pm$ 30 events, observe 263 events in the data, and therefore conclude that no excess is present in the data. Figure 2 shows a comparison of the $\mathbb{E}_{T}$ distribution for the 263 events in our candidate sample with the expected distribution from the SM backgrounds and from a LED signal with $n=2$ and $M_{D}=1 \mathrm{TeV}$. The shape of the distribution for signal events does not look significantly different than that shown here for the SM processes.

We set lower limits on the $(4+n)$-dimensional Planck scale using these results. We use PYTHIA in conjuction with the full detector simulation to generate samples of simulated graviton production, based on leading-order production cross sections calculated in Ref. [1]. We simulate the signal processes for numbers of extra dimensions between 2 and 6 and for a set of different $M_{D}$ values. The cumulative signal acceptance for our selection criteria ranges from $(9.9 \pm 1.3) \%$ to $(12.6 \pm 1.7) \%$ as a function of $n$. The acceptances are found to have no significant dependence on $M_{D}$. Contributions to the uncertainty on the acceptance include the choice of parton distribution functions $(6 \%$ relative uncertainty), possible differences in the jet energy
TABLE II. $95 \%$ C.L. limits on $M_{D}$ for $n=2-6$, with the corresponding limit on $R$, assuming toroidal compactification as described in [1].

\begin{tabular}{lcc}
\hline \hline$n$ & $M_{D}(\mathrm{TeV})$ & $R(\mathrm{~mm})$ \\
\hline 2 & $>1.18$ & $<3.5 \times 10^{-1}$ \\
3 & $>0.99$ & $<3.6 \times 10^{-6}$ \\
4 & $>0.91$ & $<1.1 \times 10^{-8}$ \\
5 & $>0.86$ & $<3.5 \times 10^{-10}$ \\
6 & $>0.83$ & $<3.4 \times 10^{-11}$ \\
\hline \hline
\end{tabular}

scale [8] between data and simulation ( $8 \%)$, the models for initial and final state radiation in the Monte Carlo simulation $(5 \%)$, and the uncertainty on the integrated luminosity of the sample $(6 \%)$. We scale the generated cross sections by a $K$ factor (the ratio of cross sections as calculated at next-to-leading order and leading order) of 1.3 [14].

We obtain the upper limit on the number of signal events in our candidate sample using a Bayesian approach with a flat prior for the number of signal events and gamma distributions for the priors for both the acceptance and the number of background events [15]. Based on 263 observed events, a SM expectation of $265 \pm 30$ events, and a combined uncertainty of $13 \%$ on the signal acceptance, we obtain an upper limit of 67 signal events at $95 \%$ confidence level (C.L.), corresponding to a cross section times acceptance of $\left(67 / 368 \mathrm{pb}^{-1}\right)=0.18 \mathrm{pb}$. We set limits on the value of $M_{D}$ for different values of $n$ based on the maximum possible number of observed signal events. The lower limits on $M_{D}$ for $n=2-6$ are shown in Table II for $K=1.3$. Assuming compactification on a torus, these limits on $M_{D}$ can easily be related to limits on the radius of the extra dimensions by Eq. (1).

This measurement places the most stringent limits from the Tevatron and in the case of $n=5$ and $n=6$, the world's best limits on $M_{D}$. The best limits for smaller values of $n$ come from the LEP combined results [16] in the $e e \rightarrow \gamma G$ channel. For the case of two extra dimensions, our upper limit of $0.35 \mathrm{~mm}$ on the size of the extra dimensions can be compared to the limit of $0.13 \mathrm{~mm}$ from a direct probe of gravity at short distances [17].

We thank the Fermilab staff and the technical staffs of the participating institutions for their vital contributions. This work was supported by the U.S. Department of Energy and National Science Foundation; the Italian Istituto Nazionale di Fisica Nucleare; the Ministry of Education, Culture, Sports, Science and Technology of Japan; the Natural Sciences and Engineering Research Council of Canada; the National Science Council of the Republic of China; the Swiss National Science Foundation; the A.P. Sloan Foundation; the Bundesministerium für Bildung und Forschung, Germany; the Korean Science and Engineering Foundation and the Korean Research Foundation; the Particle Physics and Astronomy Research Council and the Royal Society, UK; the Russian 
Foundation for Basic Research; the Comisión Interministerial de Ciencia y Tecnología, Spain; in part by the European Community's Human Potential Programme under Contract No. HPRN-CT-2002-00292; and the Academy of Finland.

[1] G. F. Giudice, R. Rattazzi, and J. D. Wells, Nucl. Phys. B544, 3 (1999); J. L. Hewett, Phys. Rev. Lett. 82, 4765 (1999); T. Han, J. D. Lykken, and R.-J. Zhang, Phys. Rev. D 59, 105006 (1999); E. A. Mirabelli, M. Perelstein, and M. E. Peskin, Phys. Rev. Lett. 82, 2236 (1999).

[2] N. Arkani-Hamed, S. Dimopoulos, and G. Dvali, Phys. Lett. B 429, 263 (1998); Phys. Rev. D 59, 086004 (1999).

[3] C. P. Burgess, AIP Conf. Proc. 743, 417 (2005); C. P. Burgess, Ann. Phys. (Berlin) 313, 283 (2004).

[4] G. Azuelos, P.-H. Beauchemin, and C. P. Burgess, J. Phys. G 31, 1 (2005); D. Atwood et al., Phys. Rev. D 63, 025007 (2000).

[5] B. Abbott et al. (D0 Collaboration), Phys. Rev. Lett. 86, 1156 (2001); D. Acosta et al. (CDF Collaboration), Phys. Rev. Lett. 89, 281801 (2002); V. M. Abazov et al. (D0 Collaboration), Phys. Rev. Lett. 90, 251802 (2003); D. Acosta et al. (CDF Collaboration), Phys. Rev. Lett. 92, 121802 (2004); V. M. Abazov et al. (D0 Collaboration), Phys. Rev. Lett. 95, 161602 (2005).

[6] D. Acosta et al. (CDF Collaboration), Phys. Rev. D 71, 032001 (2005); A. Sill et al., Nucl. Instrum. Methods Phys. Res., Sect. A 447, 1 (2000).T. Affolder et al., Nucl. Instrum. Methods Phys. Res., Sect. A 526, 249 (2004); L. Balka et al., Nucl. Instrum. Methods Phys.
Res., Sect. A 267, 272 (1988); G. Ascoli et al., Nucl. Instrum. Methods Phys. Res., Sect. A 268, 33 (1988); T. Dorigo et al., Nucl. Instrum. Methods Phys. Res., Sect. A 461, 560 (2001).

[7] We use a coordinate system where $\theta$ is the polar angle to the proton beam, $\phi$ is the azimuthal angle about this beam axis, and $\eta$ is the pseudorapidity defined as $-\ln \tan (\theta / 2)$.

[8] A. Bhatti et al., hep-ex/0510047 [Nucl. Instrum. Methods Phys. Res., Sect. A (to be published)].

[9] Missing transverse energy, $\mathbb{E}_{T}$, is defined as the magnitude of $-\sum_{i} E_{T}^{i} \hat{n}_{i}$, where $\hat{n}_{i}$ is a unit vector in the azimuthal plane that points from the beam line to the $i$ th calorimeter tower.

[10] D. Acosta et al. (CDF Collaboration), Phys. Rev. Lett. 94, 091803 (2005).

[11] T. Sjöstrand et al., Comput. Phys. Commun. 135, 238 (2001).

[12] R. Brun et al., CERN Report No. CERN-DD-78-2-REV; S. Agostinelli et al., Nucl. Instrum. Methods Phys. Res., Sect. A 506, 250 (2003).

[13] John Campbell and R. K. Ellis, Phys. Rev. D 65, 113007 (2002).

[14] P. Mathews, V. Ravindran, K. Sridhar, and W. L. van Neerven, Nucl. Phys. B713, 333 (2005); P. Mathews, V. Ravindran, and K. Sridhar, J. High Energy Phys. 10 (2005) 031.

[15] J. Heinrich et al., physics/0409129.

[16] S. Ask, Proceedings of 32nd International Conference on High Energy Physics (ICHEP, 2004) (World Scientific, Hackensack, 2005), Vol. 2, p. 1289.

[17] C. D. Hoyle et al., Phys. Rev. D 70, 042004 (2004). 